\title{
Neutrino spin relaxation in medium with stochastic characteristics
}

\author{
Maxim Dvornikov \\ Institute of Terrestrial Magnetism, Ionosphere and \\ Radiowave Propagation (IZMIRAN) \\ 142190, Troitsk, Moscow region, Russid*
}

(Dated: November 16, 2018)

\begin{abstract}
The helicity evolution of a neutrino propagating in randomly moving and polarized matter is studied. The type of the neutrino interaction with background fermions is arbitrary. We derive the equation for the description of the averaged neutrino helicity evolution. In the particular case of a $\tau$-neutrino interacting with ultrarelativistic electron-positron plasma we obtain the expression for the neutrino helicity relaxation rate in the explicit form. We study the neutrino spin relaxation in the relativistic primordial plasma. Supposing that the conversion of left-handed neutrinos into right-handed ones is suppressed at the early stages of the Universe evolution we get the upper limit on the $\tau$-neutrino mass.
\end{abstract}

PACS numbers: 14.60.Pq, 52.25.Gj

Keywords: neutrino helicity, electromagnetic fluctuations, relativistic plasma, early Universe

It is well known that if a particle, possessing the magnetic moment, interacts with the external electromagnetic field, particle's spin will rotate around a certain direction determined by this external field. The covariant description of the particle's spin precession in the external electromagnetic field was presented in Ref. 1]. Recently it was established (see Refs. 2, 3]) that weak interactions can also cause the particle's spin precession. A neutral 1/2 spin particle (e.g., a neutrino) interacting with matter via weak currents was studied in Refs. 2, 3, 4, 5]. It was found that the particle's spin precession rate depends on the velocities and polarizations of the background matter fermions. On the basis of the elaborated approach one can study various interesting phenomena such as, for instance, neutrino spin light and self-polarization effect in matter (see Refs. [6, 7] ).

In case of a neutrino interacting with medium the effect of velocities and polarizations of the background fermions on the neutrino's spin rotation is suppressed by the factor $1 / \gamma$, where $\gamma=E_{\nu} / m_{\nu}$. Thus for relativistic neutrinos the transitions between left and right polarized particles due to the matter effects vanish. In order to achieve the essential neutrino spin rotation rate one should have background fermions with very high velocities or polarizations. Such ultrarelativistic matter can exist mainly in various astrophysical environments or in the relativistic plasma of the early Universe.

Neutrinos in the early Universe were discussed for the first time in Ref. [8]. Since then a great deal of papers devoted to this topic have been published. The comprehensive review of neutrinos in cosmology was presented in Ref. [9]. The neutrino mass constraints can also be obtained from the studying of the early Universe evolution. For example, one extracts information about neutrino mass from the Cosmic Microwave Background radiation, power spectrum in large scale structure surveys as well

*Electronic address: maxdvo@izmiran.ru as Lyman $\alpha$ forest studies. The review of these issues was presented in Ref. [10].

In this paper we study neutrino spin dynamics in medium with stochastic characteristics such as number density, velocities and polarizations of background fermions. The analysis is based on the generalized relativistic invariant Bargmann-Michel-Telegdi equation which describes neutrino spin evolution in presence of general interactions. That is why the type of the neutrino interactions with medium remains arbitrary. This equation enables one to study the neutrino spin precession at arbitrary moving and polarized matter even at the absence of the external electromagnetic fields. This case can be of great importance if a neutrino is treated, for example, within the context of minimally extended standard model. The magnetic moment of such a neutrino has quite small value (see, e.g., Refs. 11, 12]),

$$
\mu_{\nu} \approx 3.2 \times 10^{-19} \mu_{B}\left(\frac{m_{\nu}}{\mathrm{eV}}\right) .
$$

The external electromagnetic field cannot effectively influence on the spin dynamics of a neutrino with such electromagnetic properties. We derive the most general equation for the description of the averaged neutrino helicity evolution. It is found that the neutrino helicity relaxation rate depends on the correlation functions of the hydrodynamical currents of background fermions. These correlators are calculated for the ulrarelativistic electronpositron plasma. The results of the paper are used for the evaluation of cosmological upper bound on the $\tau$-neutrino mass.

We start with the generalized Bargmann-MichelTelegdi equation for the neutral $1 / 2$ spin fermion (e.g., neutrino) interacting with arbitrary moving and polarized matter (see Ref. [4]),

$$
\begin{aligned}
\frac{d S^{\mu}}{d \tau} & =2 \mu\left(G^{\mu \nu} S_{\nu}-u^{\mu} G_{\alpha \beta} u_{\alpha} S_{\beta}\right) \\
& +2 \varepsilon\left(\widetilde{G}^{\mu \nu} S_{\nu}-u^{\mu} \widetilde{G}_{\alpha \beta} u_{\alpha} S_{\beta}\right),
\end{aligned}
$$


where $G_{\mu \nu}=(-\mathbf{P}, \mathbf{M})$ is the antisymmetric tensor which accounts for the matter effects, $\widetilde{G}_{\mu \nu}=(1 / 2) \varepsilon_{\mu \nu \alpha \beta} G^{\alpha \beta}=$ $(\mathbf{M}, \mathbf{P})$ is the tensor dual to $G_{\mu \nu}, \mu$ and $\varepsilon$ are the magnetic and electric dipole moments of a particle, $u^{\mu}$ is the four velocity and $S^{\mu}$ is the four dimensional spin vector. The derivative is taken with respect to the particle's proper time $\tau$.

According to the results of Ref. [4] the tensor $G_{\mu \nu}$ can be represented in the following way

$$
G_{\mu \nu}=\varepsilon_{\mu \nu \alpha \beta} g^{\alpha} u^{\beta}-\left(f_{\mu} u_{\nu}-f_{\nu} u_{\mu}\right),
$$

where the four vectors $g^{\mu}=\left(g^{0}, \mathbf{g}\right)$ and $f^{\mu}=\left(f^{0}, \mathbf{f}\right)$ depend on the four vectors of hydrodynamical currents and polarizations of medium fermions,

$$
\begin{aligned}
& g^{\mu}=\sum_{f}\left(\rho_{f}^{(1)} j_{f}^{\mu}+\xi_{f}^{(1)} \lambda_{f}^{\mu}\right), \\
& f^{\mu}=\sum_{f}\left(\rho^{(2)} j_{f}^{\mu}+\xi^{(2)} \lambda_{f}^{\mu}\right) .
\end{aligned}
$$

The constants, $\rho_{f}^{(1,2)}$ and $\xi_{f}^{(1,2)}$, are fixed by the model of the neutrino interaction with background fermions, the sum is taken over all fermionic species. It is also possible to represent the dependence of the vectors $\mathbf{M}$ and $\mathbf{P}$ on the components of the vectors $g^{\mu}$ and $f^{\mu}$ (see Ref. [4]),

$$
\begin{aligned}
\mathbf{M} & =\gamma\left\{\left(g^{0} \boldsymbol{\beta}-\mathbf{g}\right)-[\boldsymbol{\beta} \times \mathbf{f}]\right\}, \\
\mathbf{P} & =-\gamma\left\{\left(f^{0} \boldsymbol{\beta}-\mathbf{f}\right)+[\boldsymbol{\beta} \times \mathbf{g}]\right\},
\end{aligned}
$$

where $\boldsymbol{\beta}$ is the neutrino velocity. The dependence of these vectors on the velocities and polarizations of the background fermions is also presented in Ref. [4] in the explicit form.

It is convenient to rescale the vectors $\mathbf{M}$ and $\mathbf{P}$ to exclude magnetic and electric dipole moments from Eq. (2),

$$
\mathbf{M} \rightarrow \frac{\mathbf{M}}{\mu}, \quad \mathbf{P} \rightarrow \frac{\mathbf{P}}{\varepsilon} .
$$

Then we should rewrite Eq. (2) using three dimensional neutrino spin vector, $\boldsymbol{\zeta}$, which is related to the four dimensional neutrino spin vector by the following formula

$$
S^{\mu}=\left(\frac{(\zeta \mathbf{p})}{m_{\nu}}, \boldsymbol{\zeta}+\frac{\mathbf{p}(\zeta \mathbf{p})}{m_{\nu}\left(m_{\nu}+E_{\nu}\right)}\right),
$$

where $\mathbf{p}$ is the neutrino momentum. With help of Eqs. (2) and (3) we obtain the equation for the vector $\boldsymbol{\zeta}$,

$$
\frac{d \boldsymbol{\zeta}}{d t}=\frac{2}{\gamma}\left[\boldsymbol{\zeta} \times \mathbf{M}_{0}\right]-\frac{2}{\gamma}\left[\boldsymbol{\zeta} \times \mathbf{P}_{0}\right]
$$

Note that the dynamics of the neutrino spin is determined by the vectors $\mathbf{M}$ and $\mathbf{P}$ in the neutrino rest frame,

$$
\begin{aligned}
& \mathbf{M}_{0}=\gamma \boldsymbol{\beta}\left(g^{0}-\frac{1}{1+\gamma^{-1}}(\mathbf{g} \boldsymbol{\beta})\right)-\mathbf{g}, \\
& \mathbf{P}_{0}=-\gamma \boldsymbol{\beta}\left(f^{0}-\frac{1}{1+\gamma^{-1}}(\mathbf{f} \boldsymbol{\beta})\right)+\mathbf{f} .
\end{aligned}
$$

It is convenient to introduce the new vector $\boldsymbol{\Omega}=\mathbf{M}_{0}-\mathbf{P}_{0}$ and rewrite Eq. (4) in the form,

$$
\frac{d \boldsymbol{\zeta}}{d t}=\frac{2}{\gamma}[\boldsymbol{\zeta} \times \boldsymbol{\Omega}] .
$$

The neutrino spin precession is now determined by the vector $\boldsymbol{\Omega}$ which can be expressed in terms of the components of the new auxiliary four vector $A^{\mu}=\left(A^{0}, \mathbf{A}\right)$,

$$
\boldsymbol{\Omega}=\gamma \boldsymbol{\beta}\left(A^{0}-\frac{1}{1+\gamma^{-1}}(\mathbf{A} \boldsymbol{\beta})\right)-\mathbf{A} .
$$

The four vector $A^{\mu}$ also depends on the four vectors of hydrodynamical currents and polarizations of medium fermions,

$$
A^{\mu}=g^{\mu}+f^{\mu}=\sum_{f}\left(\rho_{f} j_{f}^{\mu}+\xi_{f} \lambda_{f}^{\mu}\right),
$$

where the coefficients $\rho_{f}$ and $\xi_{f}$ are related to the constants $\rho_{f}^{(1,2)}$ and $\xi_{f}^{(1,2)}$,

$$
\rho_{f}=\rho_{f}^{(1)}+\rho_{f}^{(2)}, \quad \xi_{f}=\xi_{f}^{(1)}+\xi_{f}^{(2)} .
$$

Now let us turn to the description of the neutrino helicity evolution. Basing upon Eqs. (5) and (6) one obtains the equation for particle's helicity, $h=(\boldsymbol{\beta} \boldsymbol{\zeta}) / \beta$, which has the form

$$
\frac{d h}{d t}=\frac{2}{\gamma}(\mathbf{n}[\boldsymbol{\zeta} \times \boldsymbol{\Omega}])=-\frac{2}{\gamma}(\mathbf{A}[\mathbf{n} \times \boldsymbol{\zeta}]),
$$

where $\mathbf{n}=\boldsymbol{\beta} / \beta$ is the unit vector along the neutrino velocity.

We can solve Eq. (7) for the description of the neutrino helicity evolution by means of iterations. The similar method for the description of the neutrino helicity evolution in stochastic electromagnetic fields was proposed in Ref. 13]. One substitutes the formal integral solution of Eq. (5),

$$
\boldsymbol{\zeta}(t)=\frac{2}{\gamma} \int_{0}^{t} \mathrm{~d} t^{\prime}\left[\boldsymbol{\zeta}\left(t^{\prime}\right) \times \boldsymbol{\Omega}\left(t^{\prime}\right)\right],
$$

in Eq. (77). After some uncomplicated but rather cumbersome calculations we obtain the equation for the neutrino helicity,

$$
\begin{aligned}
\frac{d h}{d t} & =-\left(\frac{2}{\gamma}\right)^{2} \int_{0}^{t} \mathrm{~d} t^{\prime}\left\{\left(\mathbf{A}_{\perp}(\mathbf{r}, t) \mathbf{A}_{\perp}\left(\mathbf{r}^{\prime}, t^{\prime}\right)\right) h\left(t^{\prime}\right)\right. \\
& \left.+\gamma\left(\boldsymbol{\zeta}_{\perp}\left(t^{\prime}\right) \mathbf{A}_{\perp}(\mathbf{r}, t)\right)\left[\beta A^{0}\left(\mathbf{r}^{\prime}, t^{\prime}\right)-A_{\|}\left(\mathbf{r}^{\prime}, t^{\prime}\right)\right]\right\} .
\end{aligned}
$$

The longitudinal, $A_{\|}=(\mathbf{n A})$, and transversal, $\mathbf{A}_{\perp}=$ $\mathbf{A}-\mathbf{n}(\mathbf{n A})$, components of the vector $\mathbf{A}$ are taken with respect to the neutrino velocity. The vectors $\mathbf{r}$ and $\mathbf{r}^{\prime}$ are the neutrino positions at the times $t$ and $t^{\prime}$. If we suppose that a neutrino propagates along a line, then $\mathbf{r}^{\prime}-\mathbf{r}=\boldsymbol{\beta}\left(t^{\prime}-t\right)$. 
Now let us discuss the average quantities in Eq. (8) that will be denoted as $\langle\ldots\rangle$. We have already mentioned that the neutrino helicity evolution is suppressed by the factor $1 / \gamma$ which is small for relativistic neutrinos. This feature can be also derived from Eq. (8). Thus, if we consider Eq. (8) to the lowest order in $1 / \gamma$, the variation of the averaged neutrino helicity turns out to be negligibly small during the correlation time of the vector $A^{\mu}$ components, because the characteristic time scale of the correlators $\left\langle A^{\mu}(\mathbf{r}, t) A^{\nu}\left(\mathbf{r}^{\prime}, t^{\prime}\right)\right\rangle$ for the high temperature plasma is very short. Let us evaluate this time scale of the correlators when a $\tau$-neutrino interacts with high temperature electron-positron plasma (this problem will be discussed in details below). The time scale is approximately equal to the mean free path of plasma particles (see, e.g., Ref. [14]), i.e. electrons and positrons,

$$
\mathfrak{t} \approx \frac{T^{2}}{\alpha_{\mathrm{em}}^{2} n \Lambda},
$$

where $T$ is the plasma temperature, $n$ in the number density in plasma, $\alpha_{\mathrm{em}}=e^{2}$ is the fine structure constant and

$$
\Lambda=\ln \left(\frac{T}{\alpha_{\mathrm{em}}} \sqrt{\frac{T}{4 \pi \alpha_{\mathrm{em}} n}}\right),
$$

is the Coulomb logarithm.

Supposing that for the relativistic plasma one has $n \approx 0.36 T^{3}$ (here we take into account that both electrons and positrons conribute to the number density) and setting $T \approx 100 \mathrm{MeV}$, we get for the mean free path the following estimate, $\mathfrak{t} \approx 5.2 \times 10^{-20} \mathrm{~s}$. On the other hand one obtains for the typical time of the averaged neutrino helicity relaxation (see Eq. (17) below), $1 / \Gamma \approx 8.6 \times 10^{-4} \mathrm{~s}$. One can see from this estimates that $\mathfrak{t} \ll 1 / \Gamma$. That is why we can consider the integrand in Eq. (8) as the product of two functions: the correlators of the vector $A^{\mu}$ components and the averaged neutrino helicity.

The vector $A^{\mu}$ is the linear combination of the hydrodynamical currents and polarizations vectors. Different components of these vectors are sure to be uncorrelated. Thus we get that the correlators of different components of the vector $A^{\mu}$ vanish. Finally one obtains more simple equation for the averaged neutrino helicity

$$
\frac{d\langle h\rangle}{d t}=-\left(\frac{2}{\gamma}\right)^{2} \int_{0}^{t} \mathrm{~d} t^{\prime}\left\langle\mathbf{A}_{\perp}(\mathbf{r}, t) \mathbf{A}_{\perp}\left(\mathbf{r}^{\prime}, t^{\prime}\right)\right\rangle\left\langle h\left(t^{\prime}\right)\right\rangle .
$$

Eq. (9) is the most general one which describes the averaged neutrino helicity evolution in presence of matter with random velocities and polarizations. Note that the type of the neutrino interaction with background fermions is not fixed yet.

Eq. (9) can be transformed to the more convenient form which will be used in the further analysis. As we have already noticed the correlations of the fields $\mathbf{A}$ are not equal to zero for a rather short period of time. Thus supposing again that the neutrino helicity is practically constant during the matter fields correlation period as well as taking into account time-translation invariance of the matter fields correlators we receive more simple differential equation for the mean neutrino helicity

$$
\frac{d\langle h\rangle}{d t}=-\Gamma\langle h\rangle,
$$

where neutrino helicity relaxation rate has the form

$$
\Gamma=\left.\left(\frac{2}{\gamma}\right)^{2} \int_{0}^{\infty} \mathrm{d} t\left\langle\mathbf{A}_{\perp}(\mathbf{r}, t) \mathbf{A}_{\perp}(0,0)\right\rangle\right|_{\mathbf{r}=\boldsymbol{\beta} t} .
$$

We can take into account only $\left\langle\mathbf{j}_{f}(\mathbf{r}, t) \mathbf{j}_{f}(0,0)\right\rangle$ correlation functions in Eq. (10) if we consider neutrino interacting with the relativistic plasma. Indeed for relativistic fermions the polarization vector is proportional to the current, $\boldsymbol{\lambda}_{f} \approx h_{f} \mathbf{j}_{f}$, where $h_{f}=\left(\boldsymbol{\beta}_{f} \boldsymbol{\zeta}_{f}\right) / \beta_{f}$ is the fermion helicity, $\boldsymbol{\beta}_{f}$ is the velocity of the reference frame in which the mean momentum of $f$-type background fermions is zero and $\boldsymbol{\zeta}_{f}$ is the mean value of the polarization vector of the $f$-type background fermions in the above mentioned reference frame. The fermion helicities and currents in a stochastic plasma take the form

$$
h_{f}=\left\langle h_{f}\right\rangle+\delta h_{f}, \quad \mathbf{j}_{f}=\left\langle\mathbf{j}_{f}\right\rangle+\delta \mathbf{j}_{f},
$$

where $\left\langle h_{f}\right\rangle=0$ and $\left\langle\mathbf{j}_{f}\right\rangle=0$ are the mean values of the corresponding quantities. The fluctuations of fermion helicities, $\delta h_{f}$, and currents, $\delta \mathbf{j}_{f}$, are taken to be small. Therefore the correlation functions like $\left\langle\boldsymbol{\lambda}_{f}(\mathbf{r}, t) \mathbf{j}_{f}(0,0)\right\rangle$ and $\left\langle\boldsymbol{\lambda}_{f}(\mathbf{r}, t) \boldsymbol{\lambda}_{f}(0,0)\right\rangle$ are next to the leading order.

The hydrodynamical currents correlation functions can be expressed in terms of the electromagnetic currents correlators for plasma consisted of charged particles. If the charges of particles in plasma are equal to $\pm e$, then

$$
\left\langle\mathbf{j}_{f}(\mathbf{r}, t) \mathbf{j}_{f}(0,0)\right\rangle=\frac{1}{e^{2}}\left\langle\mathbf{j}_{f}^{\mathrm{em}}(\mathbf{r}, t) \mathbf{j}_{f}^{\mathrm{em}}(0,0)\right\rangle,
$$

where $\mathbf{j}_{f}^{\mathrm{em}}(\mathbf{r}, t)$ is the electromagnetic current. Thus the neutrino helicity relaxation rate is expressed in the following way

$$
\begin{aligned}
\Gamma & =\left(\frac{2}{\gamma}\right)^{2} \frac{1}{e^{2}} \sum_{f} \rho_{f}^{2} \\
& \times\left.\int_{0}^{\infty} \mathrm{d} t\left\langle\mathbf{j}_{f \perp}^{\mathrm{em}}(\mathbf{r}, t) \mathbf{j}_{f \perp}^{\mathrm{em}}(0,0)\right\rangle\right|_{\mathbf{r}=\boldsymbol{\beta} t} .
\end{aligned}
$$

The correlation functions in Eq. (11) are usually expressed in terms of the Fourier transforms,

$$
\begin{aligned}
& \left\langle j_{i}^{\mathrm{em}}(\mathbf{r}, t) j_{j}^{\mathrm{em}}(0,0)\right\rangle \\
& \quad=\frac{1}{(2 \pi)^{4}} \int \mathrm{d} \omega \mathrm{d}^{3} \mathbf{k}\left(j_{i}^{\mathrm{em}} j_{j}^{\mathrm{em}}\right)_{\omega \mathbf{k}} e^{-i \omega t+i \mathbf{k r}} .
\end{aligned}
$$

The electromagnetic currents correlators can be obtained with help of the fluctuation-dissipation theorem (see Ref. 15]). The explicit form of the correlation functions is presented in Ref. [16], 


$$
\left(j_{i}^{\mathrm{em}} j_{j}^{\mathrm{em}}\right)_{\omega \mathbf{k}}=\frac{\omega^{2}}{2 \pi} \frac{1}{\exp (\omega / T)-1} \times\left\{\frac{k_{i} k_{j}}{k^{2}} \frac{\Im \mathfrak{m} \epsilon_{l}}{\left|\epsilon_{l}\right|^{2}}+\left(\delta_{i j}-\frac{k_{i} k_{j}}{k^{2}}\right)\left(1-(k / \omega)^{2}\right)^{2} \frac{\Im \mathfrak{m} \epsilon_{t}}{\left|\epsilon_{t}-(k / \omega)^{2}\right|^{2}}\right\} .
$$

The correlators are expressed in terms of the permittivity tensor components, $\epsilon_{l}$ and $\epsilon_{t}$. For the case of the ultrarelativistic plasma, $T \gg m_{f}$, where $m_{f}$ is the background fermion mass, the longitudinal and transversal components of the permittivity tensor take the form (see, e.g., Ref. 17])

$$
\begin{aligned}
& \epsilon_{l}(\mathbf{k}, \omega)=1+\frac{1}{a^{2} k^{2}}\left\{1+\frac{\omega}{2 k} \ln \left[\frac{|k-\omega|}{k+\omega}\right]+i \frac{\pi \omega}{4 k}[1+\operatorname{sign}(k-\omega)]\right\}, \\
& \epsilon_{t}(\mathbf{k}, \omega)=1-\frac{1}{2 a^{2} k^{2}} \times\left\{1-\frac{k}{2 \omega}\left(1-(\omega / k)^{2}\right) \ln \left[\frac{|k-\omega|}{k+\omega}\right]-i \frac{\pi k}{4 \omega}\left(1-(\omega / k)^{2}\right)[1+\operatorname{sign}(k-\omega)]\right\},
\end{aligned}
$$

where $a$ is the Debye length which is related to the particles densities in plasma by the following formula

$$
a^{-2}=\frac{4 \pi e^{2}}{T} \sum_{f} n_{f},
$$

and

$$
\operatorname{sign}(x)= \begin{cases}1, & x>0 \\ -1, & x<0\end{cases}
$$

is the step function.

As it was pointed out in Ref. [13], we can take into account only longitudinal component of the electromagnetic currents correlators since the imaginary part of the transversal component of the permittivity tensor [which contributes to the transversal component of the electromagnetic currents correlators, see Eq. [13)] is suppressed by the additional small factor $1 / \gamma^{2}$. Using Eqs. (11)-(15) one obtains for the neutrino helicity relaxation rate,

$$
\begin{aligned}
\Gamma & \left.\approx\left(\frac{2}{\gamma}\right)^{2} \frac{1}{e^{2}} \sum_{f} \rho_{f}^{2} \frac{\beta}{16 \pi^{2} a^{2}} \int_{-1}^{+1} \mathrm{~d}(\cos \vartheta) \sin ^{2} \vartheta \cos \vartheta \int_{0}^{\infty} \mathrm{d} k \frac{\omega^{2}}{\exp (\omega / T)-1} \frac{1}{\left|\epsilon_{l}\right|^{2}}\right|_{\omega=k \beta \cos \vartheta} \\
& \approx\left(\frac{2}{\gamma}\right)^{2} \frac{1}{e^{2}} \sum_{f} \rho_{f}^{2} \frac{T^{5}}{12 \pi^{2}(a T)^{2}} \int_{0}^{\infty} \mathrm{d} z \frac{z^{2}}{e^{z}-1} \approx 0.19 N T^{5} \frac{m_{\nu}^{2}}{E_{\nu}^{2}} \sum_{f} \rho_{f}^{2},
\end{aligned}
$$

where $N$ is the number of particles species in plasma and $\vartheta$ is the angle between vectors $\mathbf{k}$ and $\boldsymbol{\beta}$. In Eq. (16) we drop small terms like $1 / \gamma^{4}$ as well as we keep the leading terms in the fine structure constant. We also suppose the frequency $\omega$ and the wave vector $\mathbf{k}$ are connected by the relation, $\omega=(\mathbf{k} \boldsymbol{\beta})=k \beta \cos \vartheta$, that immediately follows from Eq. (11).

The neutrino helicity relaxation due to the neutrino magnetic moment interaction with the stochastic electromagnetic field was studied in Ref. 13]. The helicity relaxation rate obtained in that paper is proportional to $\mu^{2}$, where $\mu$ is the neutrino magnetic moment. One can also consider the correlations between stochastic electromagnetic fields and polarizations of the background fermions. Indeed the background fermions are polarized under the influence of the external electromagnetic field. In this case the induced polarization of the $f$-type fermions has the form,

$$
\boldsymbol{\zeta}_{f}^{(\text {ind })}=\frac{1}{n_{f}}\left(\frac{1}{g_{f}^{(e)}} \kappa_{f} \mathbf{E}+\frac{1}{g_{f}^{(m)}} \frac{\chi_{f}}{1+\chi_{f}} \mathbf{B}\right),
$$

where $\kappa_{f}$ and $\chi_{f}$ are dielectric and magnetic susceptibilities, $g_{f}^{(e)}$ and $g_{f}^{(m)}$ are the gyroelectric and gyromagnetic ratios, $n_{f}$ is the number density of the $f$-type background fermions. In the latter case the corresponding contribution to the neutrino helicity relaxation rate will be proportional to the factor $\mu \times \gamma^{-1}$.

Supposing that neutrino magnetic moment is described within a realistic model, for instance the minimally extended standard model (see Refs. 11, 12]), we obtain, e.g., for a $\tau$-neutrino (this neutrino type will be considered below) magnetic moment $\mu_{\nu_{\tau}} \approx 5.8 \times 10^{-12} \mu_{B}$ [see also Eq. (11)]. The $\tau$-neutrino mass is taken to be $18.2 \mathrm{MeV}$ 18]. If a particle has such a small magnetic moment, the contribution of the corresponding terms to the neutrino helicity relaxation rate will be negligibly small 
compared to the contribution examined in our paper.

To evaluate the mean neutrino energy in Eq. (16) we note that it can be expressed with help of the neutrino energy and number densities $\left\langle E_{\nu}\right\rangle=\varrho_{E} / n_{\nu}$. For the relativistic neutrino gas which is in equilibrium at the temperature $T$ we have

$$
\varrho_{E}=2 \int \frac{\mathrm{d}^{3} \mathbf{p}}{(2 \pi)^{3}} \frac{E_{\nu}}{\exp \left(E_{\nu} / T\right)+1} \approx 0.58 T^{4}
$$

and

$$
n_{\nu}=2 \int \frac{\mathrm{d}^{3} \mathbf{p}}{(2 \pi)^{3}} \frac{1}{\exp \left(E_{\nu} / T\right)+1} \approx 0.18 T^{3} .
$$

Finally we get for the mean neutrino energy $\left\langle E_{\nu}\right\rangle \approx$ $3.22 T$.

Let us discuss a $\tau$-neutrino propagating in ultrarelativistic plasma composed of electrons and positrons. This neutrino flavor eigenstate interacts with electronpositron plasma by means of the weak neutral currents. In this case the coefficients $\rho_{f}$ are expressed in the following way

$$
\rho_{e^{-}}=-\rho_{e^{+}}=-\frac{G_{F}}{2 \sqrt{2}}\left(1-4 \sin ^{2} \theta_{W}\right),
$$

where $G_{F}$ is the Fermi constant and $\theta_{W}$ is the Weinberg angle. Using Eqs. (16)-19) we obtain for the neutrino helicity relaxation rate,

$$
\Gamma \approx 1.16 \times 10^{3}\left(\frac{m_{\nu_{\tau}}}{10 \mathrm{MeV}}\right)^{2}\left(\frac{T}{100 \mathrm{MeV}}\right)^{3} \mathrm{~s}^{-1} .
$$

The main results of the paper can be applied, e.g., for the description of the neutrino spin relaxation in the primordial plasma. The number of right-handed neutrinos is very small at present. Indeed if one observed a right-handed neutrino, it would be a direct indication on the neutrino having a non-zero mass since the wave function of a massive particle can correspond to both left- and right-handed helicity states contrary to the case of a massless particle. However, up to now only the upper bound on the neutrino mass is experimentally established. Thus we should assert that if there were right-handed neutrinos, their number would be not very great. The neutrinos with this polarization cannot be produced in great amount at the early stages of the Universe evolution. Thus we should suppose that the process of neutrino helicity relaxation (i.e. the production of right-handed neutrinos since all neutrinos are taken to be left-handed initially) is out of the thermodynamical equilibrium, i.e. $\Gamma<H$, where $H$ is the Hubble constant. At radiation dominated era of the early Universe the Hubble constant can be approximately evaluated (see, for instance, Ref. [19]),

$$
H \approx\left(G_{N}\right)^{1 / 2} T^{2} \approx 1.24 \times 10^{3}\left(\frac{T}{100 \mathrm{MeV}}\right)^{2} \mathrm{~s}^{-1}
$$

where $G_{N}$ is the Newton's constant.

When temperature of the early Universe is equal or less than $100 \mathrm{MeV}$, the muons and antimuons densities are negligible to effectively contribute to the neutrino helicity relaxation. The densities of neutrons and protons are also small. Thus a $\tau$-neutrino can interact only with electrons and positrons at this temperature. From Eqs. (20) and (21) for $T=100 \mathrm{MeV}$ we get $m_{\nu_{\tau}}<10.2 \mathrm{MeV}$. The obtained upper limit on the $\tau$-neutrino mass is compatible with the up to date neutrino mass bounds derived in various techniques (see Ref. 18]). It should be noted that the calculations made in this paper are also consistent with the obtained upper limit. Indeed in deriving of Eq. (16) we supposed that $1 / \gamma^{4}=\left(m_{\nu} / E_{\nu}\right)^{4} \ll 1$. Eqs. (17) and (18) are valid while $m_{\nu}<T$. All these assumptions are in agreement with the temperature of the primordial plasma $(T=100 \mathrm{MeV})$ and bound on the neutrino mass $\left(m_{\nu_{\tau}} \lesssim 10 \mathrm{MeV}\right)$. It is worth mentioning that the decoupling temperature of $\tau$-neutrinos found in Ref. [20] is $\approx 3.7 \mathrm{MeV}$. Thus down to this temperature $\tau$-neutrinos are in thermal equilibrium with primeval plasma.

Although we studied the $\tau$-neutrino helicity relaxation in the early Universe, the interaction of the neutrino spin with the gravitational field (space-time curvature) was not taken into account in our work, i.e. all the calculations were performed in the minkowskian space-time. However the neutrino propagation in strong gravitational fields of the specific configurations (e.g., Kerr metric) can cause the asymmetry between neutrinos and antineutrinos. The most recent analysis of this issue is presented in Ref. 21]. In spite of the fact that the Universe is assumed to expand isotropically this effect is of great importance on the early stages of the Universe evolution when relic neutrinos interact with primordial black holes before neutrinos decoupled (see Ref. 22]). This process could influence the neutrino helicity relaxation.

It should be noted that for the first time the cosmological upper bound on the sum of all neutrinos masses was derived in Ref. [23]. One can obtain the contribution of stable light neutrinos to the matter density in the Universe and then compare the calculated value of the neutrino contribution with the observational value of total matter density. The "classical" cosmological bound (Gerstein-Zeldovich limit) is very small

$$
\sum_{i} m_{\nu_{i}}<14 \mathrm{eV}
$$

In our paper the production rate of "wrong-helicity" (i.e. right-handed) Dirac neutrinos was constrained by the Hubble parameter. For the first time the similar idea was used in Ref. 24]. It was assumed that if the population of the right-handed neutrino states in the primordial plasma is great, then they could influence the primordial nucleosynthesis by enlarging the effective number of the neutrino species.

In conclusion we note that the helicity evolution of a neutrino interacting with matter having random distri- 
butions of number density, velocities and polarizations has been studied in this paper. The basic equation was the generalized relativistic invariant Bargmann-MichelTelegdi equation. We have derived the expression for the neutrino helicity relaxation rate which depends on the correlation functions of the hydrodynamical currents of background fermions. These correlators have been evaluated for the case of the ultrarelativistic electron-positron plasma. Then we have studied $\tau$-neutrino helicity evolution in plasma of the early Universe at $T=100 \mathrm{MeV}$. Comparing the helicity relaxation rate with the Hubble constant we have obtained the upper bound on the $\tau$ - neutrino mass which turned out to be $\approx 10 \mathrm{MeV}$. This $\tau$-neutrino mass limit is consistent with modern laboratory constraints.

\section{Acknowledgments}

This research was supported by grants of Deutscher Akademischer Austausch Dienst and Russian Science Support Foundation.
[1] V. Bargmann, L. Michel, and V. L. Telegdi, Phys. Rev. Lett. 2, 435 (1959).

[2] A. M. Egorov, A. E. Lobanov, and A. I. Studenikin, in New Worlds in Astroparticle Physics, edited by A. M. Mourão, M. Pimento, and P. M. Sá (World Scientific, Singapore, 1999), p. 153, hep-ph/9902417.

[3] A. Egorov, A. Lobanov, and A. Studenikin, Phys. Lett. B 491, 137 (2000), hep-ph/9910476.

[4] A. E. Lobanov and A. I. Studenikin, Phys. Lett. B 515, 94 (2001), hep-ph/0106101.

[5] M. Dvornikov and A. Studenikin, J. High Energy Phys. 09, 016 (2002), hep-ph/0202113.

[6] A. Lobanov and A. Studenikin, Phys. Lett. B 564, 27 (2003), hep-ph/0212393.

[7] A. Lobanov and A. Studenikin, Phys. Lett. B 601, 171 (2004), astro-ph/0408026.

[8] R. A. Alpher, J. W. Follin, and R. C. Herman, Phys. Rev. 92, 1347 (1953).

[9] A. D. Dolgov, Phys. Rep. 370, 333 (2002), hep$\mathrm{ph} / 0202122$.

[10] S. M. Bilenky et al., Phys. Rep. 379, 69 (2003), hep$\mathrm{ph} / 0211462$.

[11] K. Fujikawa and R. E. Shrock, Phys. Rev. Lett. 45, 963 (1980).

[12] M. Dvornikov and A. Studenikin, Phys. Rev. D 69,
073001 (2004), hep-ph/0305206.

[13] A. Loeb and L. Stodolsky, Phys. Rev. D 40, 3520 (1989).

[14] A. I. Akhiezer et al., Plasma Electrodynamics (Nauka, Moscow, 1974), p. 37.

[15] H. B. Callen and T. A. Welton, Phys. Rev. 83, 34 (1951).

[16] pp. 518-528, in Ref. [14].

[17] E. M. Lifschitz and L. P. Pitaevskiŭ, Physical Kinetics (Nauka, Moscow, 1979), vol. 10 of Theoretical Physics, pp. 165-166.

[18] S. Eidelman et al., Phys. Lett. B 592, 1 (2004).

[19] S. Weinberg, Gravitation and Cosmology: Principles and Applications of the General Theory of Relativity (Wiley, New York, 1972), p. 534.

[20] S. Hannestad, New J. Phys. 6, 108 (2004), hepph/0404239.

[21] B. Mukhopadhyay, to appear in Mod. Phys. Lett. A, hep$\mathrm{ph} / 0505460$.

[22] P. Singh and B. Mukhopadhyay, Mod. Phys. Lett. A 18, 779 (2003).

[23] S. S. Gerstein and Y. B. Zeldovich, JETP Lett. 4, 120 (1966).

[24] S. L. Shapiro, S. A. Teukolsky, and I. Wasserman, Phys. Rev. Lett. 45, 669 (1980). 